\title{
Risk factors cannot explain the higher prevalence rates of precancerous colorectal lesions in men
}

\author{
Elisabeth Waldmann 1,2, Georg Heinze ${ }^{3}$, Arnulf Ferlitsch ${ }^{1,2}$, Irina Gess| ${ }^{1,2}$, Daniela Sallinger ${ }^{1,2}$, Philip Jeschek ${ }^{1,2}$, \\ Martha Britto-Arias ${ }^{1,2}$, Petra Salz ${ }^{1,2}$, Elisabeth Fasching ${ }^{4}$, Bernd Jilma ${ }^{5}$, Michael Kundi ${ }^{6}$, Michael Trauner ${ }^{1,2}$ \\ and Monika Ferlitsch ${ }^{*}, 1,2$

\begin{abstract}
${ }^{1}$ Division of Gastroenterology and Hepatology, Deptartment of Internal Medicine III, Medical University of Vienna, Vienna, Austria; ${ }^{2}$ Quality Assurance Working Group, Austrian Society of Gastroenterology and Hepatology (OEGGH), Vienna, Austria; ${ }^{3}$ Department of Clinical Biometry, Medical University of Vienna, Vienna, Austria; ${ }^{4}$ Main Association of the Austrian Social Insurance Institutions, Vienna, Austria; ${ }^{5}$ Institute of Clinical Pharmacology, Medical University of Vienna, Vienna, Austria and IInstitute for Environmental Hygiene, Medical University of Vienna, Vienna, Austria
\end{abstract}

Background: Prevalence of (pre)cancerous colorectal lesions are higher in men than in women, although transition rates from advanced lesions to cancer is similar in both sexes. Our aim was to investigate whether the sex-specific difference in incidence of premalignant colorectal lesions might be explained by the impact of risk factors.

Methods: A cross-sectional study analysing health check-up examinations and screening colonoscopies performed within a national quality assurance program.

Results: A total of 25409 patients were included in this study, $50.8 \%$ were women. Median age for both sexes was 60 years (interquartile range (IQR) 54-67). A multivariable model showed that risk factors mediated only 0.6 of the $10.4 \%$ gender gap in adenoma and 0.47 of the $3.2 \%$ gender gap in advanced adenoma detection rate. Smoking was the only independent risk factor with a varying sex-specific effect (men OR 1.46, Cl 1.29, 1.64, women OR 1.76, Cl 1.53, 2.06) and advanced adenomas (men OR 1.06, Cl 0.80-1.42; women OR 2.08, Cl 1.52-2.83). Independent risk factors for adenomas were BMI (OR 1.35 per IQR, Cl 1.25-1.47) and triglyceride level (OR 1.03 per IOR, Cl 1.00-1.06); for advanced adenomas physical activity (none vs regular: OR 1.54, Cl 1.182.00, occasional vs regular: OR 1.17, Cl 1.00-1.38), cholesterol level (OR 1.13 per IOR, Cl 1.02-1.25), blood glucose level (OR 1.05 per IQR, Cl 1.01-1.09) and alcohol score (OR 1.09 per IOR, Cl 1.01-1.18).

Conclusions: Risk factors cannot explain higher prevalence rates in men. Results of this study strongly underline the need for sexspecific screening recommendations.

The incidence of colorectal cancer (CRC) varies considerably depending on demographic data, genetic and lifestyle factors (Thomas and Karagas, 1987; Siegel et al, 2013). Nevertheless, recommendations for CRC screening take into account patients' sex, age and family history of cancer, but no other risk factors (Regula et al, 2006; Schmiegel et al, 2008; Nguyen et al, 2009;
Segnan et al, 2010; Ferlitsch et al, 2011). Although concepts in preventive medicine should be personalised, data regarding the impact of risk factors on the development of colorectal lesions, in general and especially in consideration of a gender effect, are still scarce. Before such personalised recommendations for CRC prevention can be developed, the possibly gender-specific role of

*Correspondence: Professor M Ferlitsch; E-mail: monika.ferlitsch@meduniwien.ac.at

Received 4 February 2016; revised 21 August 2016; accepted 6 September 2016; published online 20 October 2016

(c) 2016 Cancer Research UK. All rights reserved 0007-0920/16 
risk factors has to be clarified. Therefore, the primary aim of this study was to investigate to which extent known risk factors (Cho et al, 2004; Larsson et al, 2005; Hermann et al, 2009; Ben et al, 2012) for CRC can explain the higher prevalence of colorectal lesions in men than in women. Secondary aims were (i) to evaluate if risk factors have a differential impact on the prevalence of adenomas and advanced adenomas in men and women and (ii) to estimate how many colorectal lesions could be prevented by reducing exposure to these risk factors to recommended levels.

For this purpose we evaluated gamma GT (GGT) levels, body mass index (BMI), cholesterol levels, HDL cholesterol levels, triglyceride levels, systolic and diastolic blood pressure levels, blood glucose levels, alcohol score, the New Zealand cardiovascular risk score, physical activity, diabetes mellitus and smoking in the context of screening colonoscopy results, using a large screening population.

\section{MATERIALS AND METHODS}

Study design. This cross-sectional study considered all patients, who underwent an opportunistic health screening examination and screening colonoscopy between 2008 and 2012, where the two examinations were no $>6$ months apart.

Study population. Public-founded systematic health screening in Austria was introduced in 1974 and was adapted 2005. This opportunistic population-based program includes a comprehensive medical history, a complete physical exam, a blood test, and in the framework of CRC screening, biannual faecal occult blood tests (FOBT) starting at the age of 40 or alternatively screening colonoscopy starting at the age of 50 years. Between November 2008 and December 2012, 2688110 health screening examinations were performed in 1783591 persons.

Owing to the lack of standardised quality guidelines in screening colonoscopy in Austria, the Austrian Society for Gastroenterology and Hepatology launched together with the Federation of the Statutory Insurance Institutions and the Austrian Cancer Aid a national quality assurance project on voluntarily basis - the 'Certificate of quality in colon cancer prevention'. Details about this project were reported elsewhere (Ferlitsch et al, 2011; Bannert et al, 2012; Reinhart et al, 2013; Kozbial et al, 2014; Waldmann et al, 2015). All colonoscopy data of this study performed between November 2008 and December 2012 were assessed within this project, and comprised 80931 colonoscopies performed in 80398 persons. Data were linked exclusively for this project by irreversibly pseudonymised social insurance numbers. This resulted in 25409 linked examinations (Figure 1).

This study was IRB approved (EK 1417/2014). Written informed consent for colonoscopy data transfer to OEGGH was obtained from every patient.

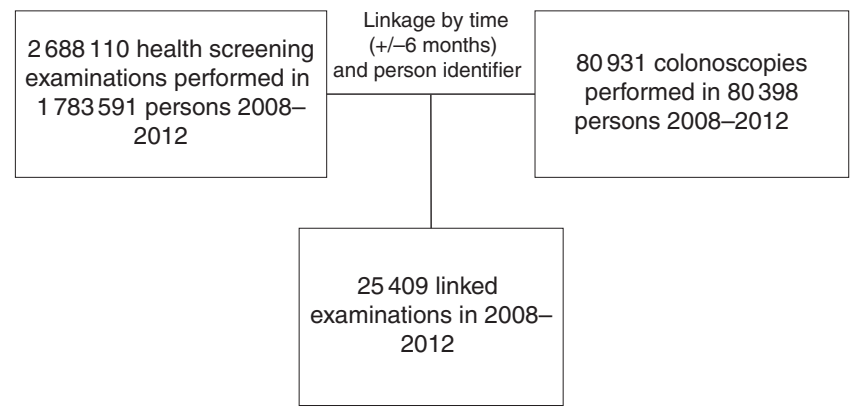

Figure 1. Flow chart of data linkage to obtain the study cohort.
Data assessment - risk factors. Alcohol intake is classified by the calculation of risk zones by Barbor et al (2001), assessed on the basis of a 40-point score questionnaire. Risk zone + is defined by $5-15$ points in women and $8-15$ points in men, risk zone ++ by $16-19$ points in both men and women, and risk zone +++ by $20-40$ points. The cardiovascular risk was assessed by the New Zealand risk score (New Zealand Primary Care Handbook 2012, which comprises risk levels grouped in four risk classes (low: $<10 \%$ absolute risk, moderate: $10-15 \%$, high $15-20 \%$ and very high $>20 \%$ ) for the chance of a cardiovascular event within the next 5 years. Physical activity is reported as either 'none', 'occasional' or 'regular', whereby occasional physical activity is defined as consumption of 150 calories per day or 1000 calories per week (NHLBI Obesity Education Initiative: The Practical Guide. Identification, Evaluation and Treatment of Overweight and Obesity in Adults, 2000).

Outcomes. As colonoscopy outcomes, we considered (i) histologically confirmed adenomas and (ii) histologically confirmed advanced adenomas, which were defined as adenomas with highgrade dysplasia, villous or tubulovillous histology or adenomas $\geq 1 \mathrm{~cm}$. If more than one lesion was detected, details on the most advanced lesion were assessed.

Statistical analysis. Continuous variables are described by median and quartiles, and categorical variables by absolute frequencies and percentages. Logistic regression was used to assess the association of detection of adenomas and detection of advanced adenomas with age, sex and risk factors. Continuous risk factors were modelled using restricted cubic splines and then reported as contrasts between quartile groups. Single-risk-factor age-adjusted models were estimated to describe association of each risk factor with outcomes. These models included an interaction of the risk factor with sex to obtain separate odds ratios for men and women, and to compare the risk for men and women at different risk factor levels. Multivariable risk-factor models were developed by backward elimination of model terms at $P>0.2$.

Mediation analysis of the gender effect (MGE) on outcomes by risk factors was conducted by evaluating the absolute and relative reduction in the difference of the average age-adjusted sex-specific predicted probabilities (the 'gender gap') after accounting for all important risk factors in the multivariable models.

Population attributable fractions (PAFs) express the proportion of adenomas or advanced adenomas that are attributable to unfavourable exposure to risk factors. PAFs were computed by the formula $P A F=\sum_{i} y_{i}-\hat{p}_{i}^{*} / \sum_{i} y_{i}$ where $y_{i}$ is the outcome of individual $i$ and $\hat{p}_{i}^{*}$ denotes the multivariable-model-based counterfactual predicted probability of that individual if unfavourably high or low values of a risk factor were replaced by the upper or lower limits, respectively, of the recommended reference ranges. PAFs were computed assuming that unfavourable values of risk factors were reverted to their respective recommended ranges; this was first assumed for each risk factor separately and then for all risk factors simultaneously. Ninetyfive per cent confidence intervals for MGE and PAF were computed by the bootstrap- $t$ method, generating 200 resamples with replacement of the original data set, and recomputing MGE and PAF from each resample to obtain s.e.'s for MGE and PAF. SAS V9.4 (2014, SAS Institute Inc., Cary, NC, USA) and R (Version 3.1.2, 2014, R Core Team, Vienna, Austria) with package rms (F. Harrell Jr, rms: Regression Modelling Strategies. R package version 4.1-3, 2014) were used for statistical analysis. 


\section{RESULTS}

This study included 25409 patients (49.2 men and 50.8\% women) with a median age of 60 years (IQR 54-67). During colonoscopy, the caecum was reached in $96.3 \%$. In $34.5 \%$ $(n=8773)$ of colonoscopies at least one polyp was detected, in $19.4 \%(n=4927)$ at least one adenoma and in 5.6\% $(n=1410)$ at least one advanced adenoma. In 65.3\% $(n=16599)$ of colonoscopies no lesion was found. Overall ADR was $24.7 \%$ in men and $14.3 \%$ in women, and the corresponding overall AADR were $7.2 \%$ and $4.0 \%$, respectively. In men, 143 (1.14\%) CRCs were detected, whereas this was the case in 91 women $(0.71 \%)$. Table 1 summarises ADR, AADR and CRC detection rates per age groups (quartiles). Median values and IQR for all investigated risk factors in men and women are summarised in Table 2.

Risk factors and adenomas. Table 3 a summarises age-adjusted odds ratios of risk factors for adenomas and Table $3 \mathrm{~b}$ for advanced adenomas in men and women. The lifestyle factors obesity $(P<0.0001)$, smoking $(P<0.001)$, low physical activity

Table 1. Colonoscopy outcomes by age and by sex, $N(\%)$

\begin{tabular}{|l|l|c|r|}
\hline Outcome & Age (years) & \multicolumn{1}{|c|}{ Males } & \multicolumn{1}{c|}{ Females } \\
\hline Adenomas & Q1: $\leqslant 54$ & $556(16.8 \%)$ & $340(10.1 \%)$ \\
& Q2: $55-60$ & $726(23.2 \%)$ & $439(13.2 \%)$ \\
& Q3: 61-67 & $899(29.8 \%)$ & $541(16.7 \%)$ \\
& Q4: $\geqslant 68$ & $906(29.7 \%)$ & $520(17.4 \%)$ \\
\hline Advanced adenomas & Q1: $\leqslant 54$ & $152(4.59 \%)$ & $91(2.72 \%)$ \\
& Q2: $55-60$ & $188(6.02 \%)$ & $122(3.68 \%)$ \\
& Q3: 61-67 & $271(8.99 \%)$ & $137(4.22 \%)$ \\
& Q4: $\geqslant 68$ & $285(9.34 \%)$ & $164(5.48 \%)$ \\
\hline Colorectal cancers & Q1: $\leqslant 54$ & $28(0.85 \%)$ & $11(0.33 \%)$ \\
& Q2: $55-60$ & $27(0.86 \%)$ & $13(0.39 \%)$ \\
& Q3: 61-67 & $37(1.23 \%)$ & $36(1.11 \%)$ \\
& Q4: $\geqslant 68$ & $51(1.67 \%)$ & $31(1.04 \%)$ \\
\hline
\end{tabular}

(men $P<0.001$ and women $P=0.020$ ), diabetes (men $P=0.032$ and women $P=0.033$ ) and a high cardiovascular risk score $(P<0.001)$ had a significant influence on presence of colorectal adenomas in both men and women. High systolic and diastolic blood pressure levels increased the prevalence in men only (men $P=0.002$ and $P=0.011$, and women $P=1.000$ and $P=0.592$ ). Interestingly, high alcohol intake was not associated with increased risk for adenomas for both sexes (men $P=0.453$ and women $P=0.894$ ), whereas high GGT levels increased the risk for men $(P<0.001)$ and high triglyceride levels the risk for both genders $($ men $<0.001$ and women 0.017). Notably, no risk factor was associated with the presence of adenomas in women only.

Risk factors and advanced adenomas. In both sexes, risk for development of advanced adenomas was significantly associated with the lifestyle factors obesity $(P<0.001)$, smoking $(P<0.001)$, low physical activity $(P<0.001)$, diabetes (men $P=0.002$, women $P=0.037)$ and cardiovascular risk score $(P<0.001)$. Interestingly, high GGT levels increased the risk for advanced adenomas in women only (men $P=0.299$ and women $P<0.003$ ). High systolic blood pressure impacted on the risk for men (men $P=0.011$ and women $P=1.000$ ); however, diastolic blood pressure was unrelated with advanced adenomas (men $P=0.257$ and women $P=0.074$ ).

Independent predictors for adenomas and advanced adenomas. Multivariable analysis identified smoking as the only independent risk factor - besides age and gender - simultaneously associated with adenomas (men OR 1.46; CI 1.29-1.64; women OR 1.76, CI 1.53-2.02) and advanced adenomas (men 1.06, CI 0.80-1.42; women OR 2.08, CI 1.52-2.83) in men and women, and revealed a significantly stronger impact of smoking in women (Figure $2 \mathrm{~A}$ and $\mathrm{B})$. Further, independent risk factors for adenomas were triglyceride level (OR 1.03 per IQR, CI 1.00-1.06), BMI (OR 1.35 per IQR, CI 1.25-1.47) and diastolic blood pressure (OR 1.02 per IQR, CI 0.94-1.12); for advanced adenomas physical activity (none vs regularly: OR 1.54, CI 1.18-2.00, occasional vs regularly: OR 1.17, CI 1.00-1.38), GGT (OR 1.05 IQR, CI 0.85-1.29), cholesterol level

Table 2. Distribution of risk factors considered in analysis

\begin{tabular}{|c|c|c|c|c|c|c|}
\hline Risk factor (unit) & $\begin{array}{l}\text { Reference } \\
\text { value }\end{array}$ & $\begin{array}{c}\text { Males } \\
\quad N\end{array}$ & $\begin{array}{c}\text { Males } \\
\text { Median (IQR) } \\
\text { or } N(\%)\end{array}$ & $\begin{array}{c}\text { Females } \\
N\end{array}$ & $\begin{array}{l}\text { Females } \\
\text { Median (IQR) } \\
\text { or } N(\%)\end{array}$ & $\begin{array}{c}\text { Total } \\
\text { Median (IQR) } \\
\text { or } N(\%)\end{array}$ \\
\hline Age (years) & - & 12505 & $60(54,67)$ & 12904 & $60(54,67)$ & $60(54,67)$ \\
\hline BMI $\left(\mathrm{kg} \mathrm{m}^{-2}\right)$ & $18.5-25.0$ & 12191 & $26.8(24.7,29.4)$ & 12606 & $25.6(23.0,29.0)$ & $26.3(23.9,29.2)$ \\
\hline Triglyceride level $\left(\mathrm{mg} \mathrm{dl}^{-1}\right)$ & $<150$ & 12479 & $115(82,167)$ & 12776 & $101(74,139)$ & $107(78,152)$ \\
\hline Systolic blood pressure $(\mathrm{mm} \mathrm{Hg})$ & $<140$ & 12503 & $134(125,145)$ & 12904 & $130(120,143)$ & $130(120,145)$ \\
\hline Diastolic blood pressure $(\mathrm{mm} \mathrm{Hg})$ & $<90$ & 12503 & $80(80,90)$ & 12904 & $80(75,86)$ & $80(77,88)$ \\
\hline Blood glucose level (mg dl ${ }^{-1}$ ) & 74-109 & 12503 & $97(88,107)$ & 12904 & $93(85,102)$ & $95(86,105)$ \\
\hline Diabetes mellitus & - & 12505 & $1070(8.6 \%)$ & 12904 & $743(5.8 \%)$ & $1813(7.1 \%)$ \\
\hline $\begin{array}{l}\text { Physical activity } \\
\text { None } \\
\text { Occasional } \\
\text { Regular }\end{array}$ & $\begin{array}{l}- \\
- \\
-\end{array}$ & 12505 & $\begin{array}{c}883(7.1) \\
4,776(38.2) \\
6,846(54.7)\end{array}$ & 12904 & $\begin{array}{c}1044(8.1) \\
5077(39.3) \\
6783(54.7)\end{array}$ & $\begin{array}{c}1927(7.6) \\
9843(38.8) \\
13629(53.6)\end{array}$ \\
\hline Smoking & - & 12505 & $1706(13.6 \%)$ & 12904 & $1687(13.1 \%)$ & $3393(13.4 \%)$ \\
\hline
\end{tabular}


Table 3a. Single-factor age-adjusted odds ratios of risk factors for adenomas

\begin{tabular}{|c|c|c|c|c|c|c|}
\hline Risk factor & $\begin{array}{c}\text { Quartile (Q) } \\
\text { or group }\end{array}$ & $\begin{array}{c}\text { Males } \\
\text { Odds ratios } \\
(95 \% \mathrm{Cl})\end{array}$ & $\begin{array}{l}\text { Males } \\
P \text {-value for } \\
\text { trend }\end{array}$ & $\begin{array}{c}\text { Females } \\
\text { Odds ratios } \\
(95 \% \mathrm{Cl})\end{array}$ & $\begin{array}{l}\text { Females } \\
P \text {-value for } \\
\text { trend }\end{array}$ & $\begin{array}{c}\text { Males vs females } \\
\text { Odds ratios } \\
(95 \% \mathrm{Cl})\end{array}$ \\
\hline GGT & $\begin{array}{l}\text { Q4: 41-192 } \\
\text { Q3: } 26-40 \\
\text { Q2: } 18-25 \\
\text { Q1: } 8-17\end{array}$ & $\begin{array}{c}1.27(1.12,1.44) \\
1.15(1.02,1.29) \\
1.05 \text { (0.94, } 1.17) \\
\text { (Ref.) }\end{array}$ & $<0.001$ & $\begin{array}{c}1.07(0.93,1.23) \\
1.04(0.93,1.17) \\
1.04 \text { (0.98, } 1.11) \\
\text { (Ref.) }\end{array}$ & 0.126 & $\begin{array}{l}2.07(1.80,2.39) \\
1.92(1.70,2.16) \\
1.76(1.55,1.99) \\
1.75(1.51,2.02)\end{array}$ \\
\hline $\mathrm{BMI}$ & $\begin{array}{c}\text { Q4: } 29.3-39.9 \\
\text { Q3: } 26.4-29.2 \\
\text { Q2: } 24-26.3 \\
\text { Q1: } 18.9-23.9\end{array}$ & $\begin{array}{c}1.64(1.45,1.85) \\
1.32(1.19,1.48) \\
1.09(0.99,1.20) \\
\text { (Ref.) }\end{array}$ & $<0.001$ & $\begin{array}{c}1.33(1.17,1.52) \\
1.22(1.10,1.34) \\
1.05(0.98,1.13) \\
\text { (Ref.) }\end{array}$ & $<0.001$ & $\begin{array}{l}2.10(1.83,2.39) \\
1.86(1.67,2.08) \\
1.77(1.56,2.01) \\
1.71(1.49,1.97)\end{array}$ \\
\hline Cholesterol & $\begin{array}{l}\text { Q4: } 246-329 \\
\text { Q3: } 218-245 \\
\text { Q2: 192-217 } \\
\text { Q1: } 127-191\end{array}$ & $\begin{array}{l}1.07(0.96,1.20) \\
1.05(0.96,1.15) \\
1.02(0.97,1.07)\end{array}$ & 0.279 & $\begin{array}{l}1.10(0.96,1.25) \\
1.12(1.00,1.26) \\
1.05(0.96,1.15)\end{array}$ & 0.083 & $\begin{array}{l}1.99(1.76,2.26) \\
1.91(1.71,2.14) \\
1.98(1.75,2.24) \\
2.04(1.79,2.34)\end{array}$ \\
\hline HDL cholesterol & $\begin{array}{l}\text { Q4: } 71-108 \\
\text { Q3: 59-70 } \\
\text { Q2: } 48-58 \\
\text { Q1: } 29-47\end{array}$ & $\begin{array}{c}0.81(0.72,0.92) \\
0.85(0.78,0.92) \\
0.92 \text { (0.87, } 0.97) \\
\text { (Ref.) }\end{array}$ & 0.003 & $\begin{array}{c}0.92(0.80,1.06) \\
0.94(0.83,1.06) \\
0.96(0.85,1.08) \\
\text { (Ref.) }\end{array}$ & 0.433 & $\begin{array}{l}1.78(1.55,2.05) \\
1.82(1.64,2.03) \\
1.92(1.70,2.17) \\
2.01(1.74,2.32)\end{array}$ \\
\hline Systolic blood pressure & $\begin{array}{l}\text { Q4: } 146-187 \\
\text { Q3: } 131-145 \\
\text { Q2: 121-130 } \\
\text { Q1: } 100-120\end{array}$ & $\begin{array}{l}1.24(1.10,1.38) \\
1.14(1.04,1.26) \\
1.11(1.03,1.19) \\
1 \text { (Ref.) }\end{array}$ & 0.002 & $\begin{array}{c}1.02(0.89,1.17) \\
0.98(0.88,1.10) \\
1.00(0.93,1.07) \\
1 \text { (Ref.) }\end{array}$ & 1.000 & $\begin{array}{l}2.14(1.86,2.45) \\
2.04(1.82,2.29) \\
1.96(1.74,2.19) \\
1.76(1.55,2.00)\end{array}$ \\
\hline Diastolic blood pressure & $\begin{array}{l}\text { Q4: } 89-110 \\
\text { Q3: } 81-88 \\
\text { Q2: 78-80 } \\
\text { Q1: } 60-77\end{array}$ & $\begin{array}{l}1.19(1.06,1.34) \\
1.08(0.98,1.20) \\
1.07(0.97,1.17) \\
1 \text { (Ref.) }\end{array}$ & 0.011 & $\begin{array}{l}1.04(0.91,1.19) \\
0.97(0.86,1.08) \\
0.95(0.87,1.05) \\
\quad 1 \text { (Ref.) }\end{array}$ & 0.592 & $\begin{array}{l}2.02(1.76,2.32) \\
1.97(1.77,2.20) \\
1.98(1.77,2.21) \\
1.76(1.53,2.03)\end{array}$ \\
\hline Blood glucose level & $\begin{array}{l}\text { Q4: 106-193 } \\
\text { Q3: 96-105 } \\
\text { Q2: 87-95 } \\
\text { Q1: 65-86 }\end{array}$ & $\begin{array}{l}1.23(1.10,1.37) \\
1.11(1.04,1.19) \\
1.03(0.98,1.09) \\
1 \text { (Ref.) }\end{array}$ & $<0.001$ & $\begin{array}{l}1.16(1.01,1.33) \\
1.09(1.00,1.18) \\
1.03(0.98,1.09) \\
1 \text { (Ref.) }\end{array}$ & 0.003 & $\begin{array}{l}1.99(1.74,2.29) \\
1.93(1.75,2.13) \\
1.89(1.69,2.12) \\
1.89(1.67,2.13)\end{array}$ \\
\hline Physical activity & $\begin{array}{c}\text { None } \\
\text { Occasionally } \\
\text { Regularly }\end{array}$ & $\begin{array}{l}1.36(1.16,1.59) \\
1.22(1.12,1.33) \\
1 \text { (Ref.) }\end{array}$ & $<0.001$ & $\begin{array}{l}1.24(1.03,1.48) \\
1.08 \text { (0.97, 1.20) } \\
1 \text { (Ref.) }\end{array}$ & 0.020 & $\begin{array}{l}2.06(1.63,2.60) \\
2.12(1.88,2.39) \\
1.87(1.68,2.09)\end{array}$ \\
\hline Diabetes mellitus & $\begin{array}{l}\text { Yes } \\
\text { No }\end{array}$ & $\begin{array}{l}1.17 \text { (1.01, 1.34) } \\
1 \text { (Ref.) }\end{array}$ & 0.032 & $\begin{array}{c}1.24(1.02,1.50) \\
1 \text { (Ref.) }\end{array}$ & 0.033 & $\begin{array}{l}1.86(1.46,2.36) \\
1.97(1.80,2.17)\end{array}$ \\
\hline Smoking & $\begin{array}{l}\text { Yes } \\
\text { No }\end{array}$ & $\begin{array}{c}1.47 \text { (1.31, 1.65) } \\
\quad 1 \text { (Ref.) }\end{array}$ & $<0.001$ & $\begin{array}{l}1.70(1.48,1.95) \\
\quad 1 \text { (Ref.) }\end{array}$ & $<0.001$ & $\begin{array}{l}1.74(1.46,2.07) \\
2.02(1.83,2.22)\end{array}$ \\
\hline
\end{tabular}

(OR 1.13 per IQR, CI 1.02-1.25), blood glucose level (OR 1.05 per IQR, CI 1.01-1.09) and alcohol score (OR 1.09 per IQR, CI 1.011.18; Table 4).

Gender effect on ADR and AADR. The non-mediated ageadjusted gender gap in ADR was 10.4 percentage points, meaning that on average, a man's ADR was 10.4 percentage points higher than that of a women of the same age. After adjusting for all independent risk factors in the multivariable model, this gender gap reduced to 9.8 percentage points, implying that only $8.6 \%$ (95\% CI: $5.4-11.8 \%$ on a relative scale) of the gender effect is mediated by the investigated risk factors (Table 5).
The age-adjusted gender gap in AADR was 3.17 percentage points. After multivariable analysis taking all independent risk factors into account, the gender gap was still 2.70 percentage points, meaning that $14.2 \%$ ( $95 \%$ CI: -8.5 to $36.9 \%$ ) of the gender effect is mediated by the risk factors (Table 5).

Population attributable fractions. Unfavourable exposure to any of the independent risk factors for adenomas was responsible for $16.7 \%$ (95\% CI, 13.9-19.4\%) of the detected adenomas and for $13.2 \%$ (95\% CI, 8.1-18.2\%) of the detected advanced adenomas. Table 6 summarises the fractions of the observed ADR and AADR attributable to each risk factor separately. The highest PAFs were estimated for smoking (adenomas 4.75\%, 95\% CI 3.66-5.84\%; advanced adenomas 6.37\%, 95\% CI 4.16-8.59\%), cardiovascular risk 
Table 3b. Single-factor age-adjusted odds ratios of risk factors for advanced adenomas

\begin{tabular}{|c|c|c|c|c|c|c|}
\hline Risk factor & $\begin{array}{l}\text { Quartile } \\
\text { (Q) or } \\
\text { group }\end{array}$ & $\begin{array}{c}\text { Males } \\
\text { Odds ratios } \\
(95 \% \mathrm{Cl})\end{array}$ & $\begin{array}{l}\text { Males } \\
P \text {-value for } \\
\text { trend }\end{array}$ & $\begin{array}{l}\text { Females } \\
\text { Odds ratios } \\
(95 \% \mathrm{Cl})\end{array}$ & $\begin{array}{l}\text { Females } \\
P \text {-value for } \\
\text { trend }\end{array}$ & $\begin{array}{l}\text { Males vs females } \\
\text { Odds ratios } \\
(95 \% \mathrm{Cl})\end{array}$ \\
\hline GGT & $\begin{array}{l}\text { Q4: } 41-192 \\
\text { Q3: } 26-40 \\
\text { Q2: } 18-25 \\
\text { Q1: } 8-17\end{array}$ & $\begin{array}{l}1.12(0.91,1.38) \\
1.00(0.83,1.21) \\
0.96 \text { (0.81, 1.14) } \\
1 \text { (Ref.) }\end{array}$ & 0.299 & $\begin{array}{l}1.15(0.89,1.48) \\
1.07(0.88,1.30) \\
0.91 \text { (0.82, 1.02) } \\
1 \text { (Ref.) }\end{array}$ & 0.003 & $\begin{array}{l}1.78(1.39,2.28) \\
1.71(1.39,2.11) \\
1.93(1.55,2.40) \\
1.83(1.44,2.33)\end{array}$ \\
\hline BMI & $\begin{array}{c}\text { Q4: } 29.3- \\
39.9 \\
\text { Q3: } 26.4- \\
29.2 \\
\text { Q2: } 24-26.3 \\
\text { Q1: } 18.9- \\
23.9\end{array}$ & $\begin{array}{c}1.58(1.28,1.95) \\
1.34(1.10,1.61) \\
1.12(0.94,1.33) \\
1 \text { (Ref.) }\end{array}$ & $<0.001$ & $\begin{array}{c}1.18(0.93,1.49) \\
1.19(0.99,1.43) \\
1.10(0.96,1.25) \\
1 \text { (Ref.) }\end{array}$ & $<0.001$ & $\begin{array}{l}2.10(1.67,2.63) \\
1.75(1.45,2.12) \\
1.59(1.28,1.98) \\
1.57(1.22,2.01)\end{array}$ \\
\hline Cholesterol & $\begin{array}{l}\text { Q4: } 246-329 \\
\text { Q3: } 218-245 \\
\text { Q2: } 192-217 \\
\text { Q1: } 127-191\end{array}$ & $\begin{array}{l}1.17(0.98,1.41) \\
1.16(0.99,1.35) \\
1.07(0.99,1.17) \\
1 \text { (Ref.) }\end{array}$ & 0.120 & $\begin{array}{l}1.12(0.88,1.43) \\
1.15(0.93,1.42) \\
1.08 \text { (0.92, 1.27) } \\
1 \text { (Ref.) }\end{array}$ & 0.272 & $\begin{array}{l}1.97(1.58,2.44) \\
1.89(1.56,2.29) \\
1.87(1.51,2.31) \\
1.88(1.48,2.39)\end{array}$ \\
\hline Triglycerides & $\begin{array}{l}\text { Q4: } 153-425 \\
\text { Q3: } 108-152 \\
\text { Q2: 79-107 } \\
\text { Q1: } 38-78\end{array}$ & $\begin{array}{l}1.41(1.18,1.69) \\
1.15(1.00,1.33) \\
1.01 \text { (0.89, 1.15) } \\
1 \text { (Ref.) }\end{array}$ & $<0.001$ & $\begin{array}{l}1.14(0.89,1.47) \\
1.22(1.00,1.47) \\
1.24(1.05,1.47) \\
1 \text { (Ref.) }\end{array}$ & 0.400 & $\begin{array}{l}2.21(1.72,2.83) \\
1.69(1.40,2.02) \\
1.45(1.17,1.79) \\
1.78(1.41,2.25)\end{array}$ \\
\hline Systolic blood pressure & $\begin{array}{l}\text { Q4: } 146-187 \\
\text { Q3: } 131-145 \\
\text { Q2: } 121-130 \\
\text { Q1: } 100-120\end{array}$ & $\begin{array}{l}1.31(1.09,1.59) \\
1.21(1.02,1.42) \\
1.09(0.96,1.24) \\
1 \text { (Ref.) }\end{array}$ & 0.011 & $\begin{array}{l}0.90(0.71,1.16) \\
0.86(0.70,1.06) \\
0.96(0.84,1.08) \\
1 \text { (Ref.) }\end{array}$ & 1.000 & $\begin{array}{l}2.26,1.78,2.87) \\
2.17(1.77,2.66) \\
1.77(1.45,2.17) \\
1.55(1.25,1.94)\end{array}$ \\
\hline Diastolic blood pressure & $\begin{array}{l}\text { Q4: } 89-110 \\
\text { Q3: } 81-88 \\
\text { Q2: 78-80 } \\
\text { Q1: } 60-77\end{array}$ & $\begin{array}{l}1.13(0.93,1.37) \\
0.98(0.83,1.16) \\
0.94(0.81,1.10) \\
1 \text { (Ref.) }\end{array}$ & 0.257 & $\begin{array}{l}1.21(0.95,1.54) \\
1.03(0.85,1.25) \\
0.89(0.76,1.05) \\
1 \text { (Ref.) }\end{array}$ & 0.074 & $\begin{array}{l}1.70(1.35,2.15) \\
1.75(1.44,2.12) \\
1.94(1.59,2.37) \\
1.83(1.44,2.32)\end{array}$ \\
\hline New Zealand cardiovascular risk score & $\begin{array}{c}5-7 \\
4 \\
3 \\
1\end{array}$ & $\begin{array}{l}2.41(1.82,3.19) \\
1.70(1.43,2.01) \\
1.42(1.27,1.59) \\
1 \text { (Ref.) }\end{array}$ & $<0.001$ & $\begin{array}{l}2.43(1.58,3.75) \\
1.70(1.31,2.21) \\
1.43(1.20,1.70) \\
1 \text { (Ref.) }\end{array}$ & $<0.001$ & $\begin{array}{l}1.57(1.01,2.42) \\
1.57(1.21,2.04) \\
1.57(1.29,1.91) \\
1.58(1.29,1.94)\end{array}$ \\
\hline Physical activity & $\begin{array}{c}\text { None } \\
\text { Occasionally } \\
\text { Regularly }\end{array}$ & $\begin{array}{l}1.75(1.38,2.24) \\
1.39 \text { (1.21, 1.61) } \\
1 \text { (Ref.) }\end{array}$ & $<0.001$ & $\begin{array}{l}1.63(1.23,2.17) \\
1.06(0.88,1.29) \\
1 \text { (Ref.) }\end{array}$ & $<0.001$ & $\begin{array}{l}1.80(1.26,2.58) \\
2.20(1.78,2.71) \\
1.68(1.38,2.04)\end{array}$ \\
\hline Diabetes mellitus & $\begin{array}{l}\text { Yes } \\
\text { No }\end{array}$ & $\begin{array}{c}1.40 \text { (1.13, 1.73) } \\
1 \text { (Ref.) }\end{array}$ & 0.002 & $\begin{array}{c}1.41 \text { (1.02, 1.93) } \\
1 \text { (Ref.) }\end{array}$ & 0.037 & $\begin{array}{l}1.84(1.25,2.71) \\
1.85(1.57,2.18)\end{array}$ \\
\hline Smoking & $\begin{array}{l}\text { Yes } \\
\text { No }\end{array}$ & $\begin{array}{c}1.42 \text { (1.17, 1.71) } \\
1 \text { (Ref.) }\end{array}$ & $<0.001$ & $\begin{array}{c}2.01 \text { (1.60, 2.53) } \\
1 \text { (Ref.) }\end{array}$ & $<0.001$ & $\begin{array}{l}1.40(1.05,1.87) \\
1.99(1.68,2.36)\end{array}$ \\
\hline
\end{tabular}

score (adenomas 3.15\%, 95\% CI 2.43-3.87\%; advanced adenomas $5.72 \%$, 95\% CI 3.88-7.57\%) and HDL cholesterol (adenomas 2.94\%, 95\% CI 1.21-4.67\%; advanced adenomas 6.05\%, 95\% CI $2.69-9.42 \%)$.

\section{CONCLUSIONS}

The present study investigated the impact of cardiovascular and lifestyle factors on the prevalence of colorectal adenomas and advanced adenomas in a large screening colonoscopy cohort of
25409 individuals; and whether the impact of these risk factors might explain the higher prevalence rates of premalignant colorectal lesions in men. Overall ADR in this study cohort was $24.7 \%$ in men and $14.3 \%$ in women; overall AADR was $7.2 \%$ and $4.0 \%$, respectively. Interestingly, on a relative scale only $8.6 \%$ of the $10.4 \%$ points of gender age gap in ADR and $14.2 \%$ of the $0.47 \%$ point age gap in AADR was mediated by the investigated risk factors. Therefore, higher prevalence rates could not explained by the impact of cardiovascular and lifestyle risk factors, and male sex seems to have the strongest impact on the prevalence of colorectal lesions. 
A

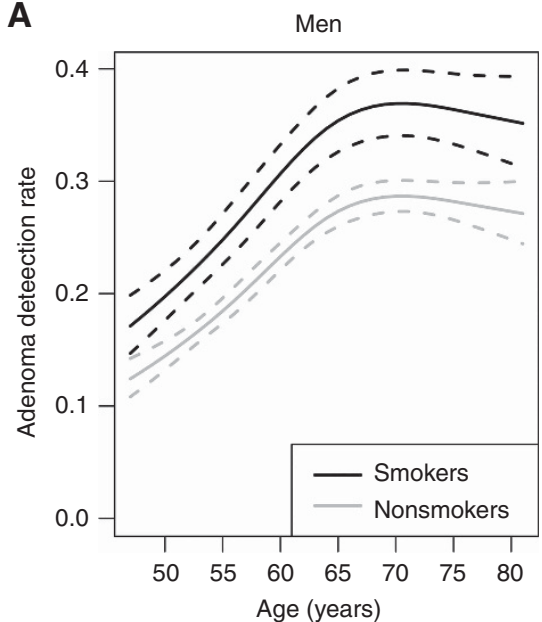

B

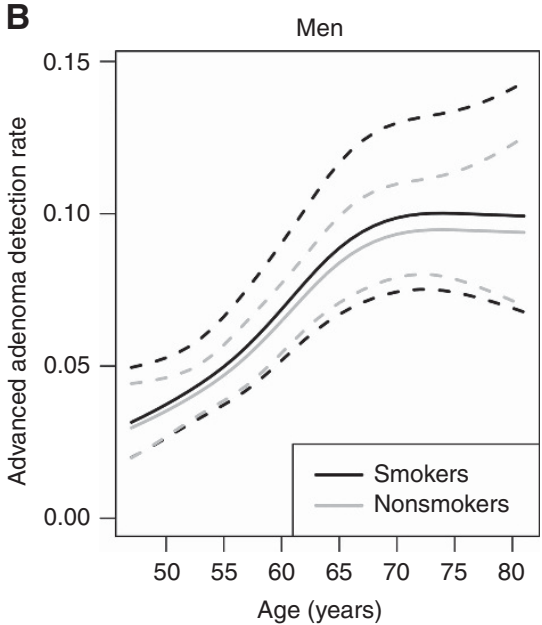

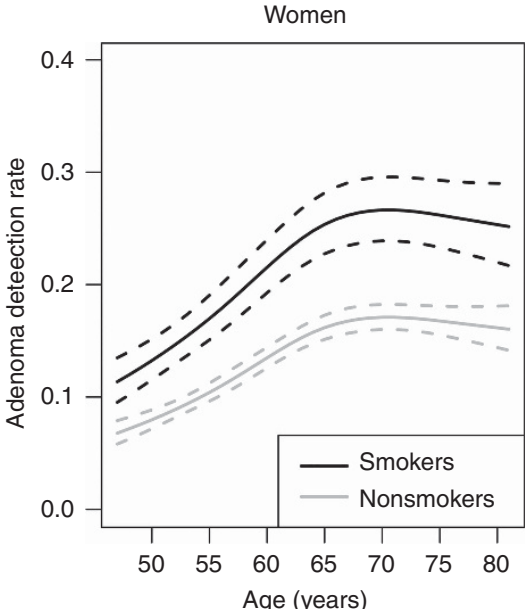

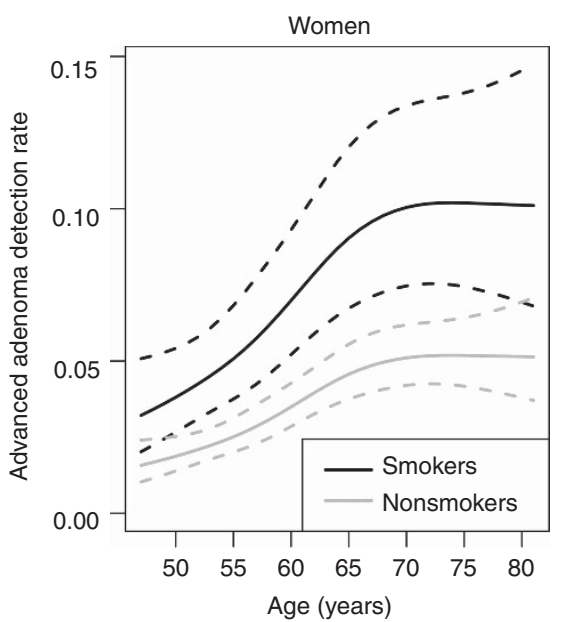

Figure 2. (A) Estimated adenoma detection rate for smoking and non-smoking men and women (solid lines) and $95 \%$ confidence intervals (dashed lines). The estimated adenoma detection rates are adjusted for triglyceride level, bodymassindex and diastolic blood pressure.

(B) Estimated advanced adenoma detection rate for smoking and non-smoking men and women (solid lines) and $95 \%$ confidence intervals (dashed lines). The estimated advanced adenoma detection rates are adjusted for physical activity, GGT=cholesterol level, blood glucose level and alcohol score.

Smoking has shown to be strongly associated with precancerous colorectal lesions in men and women (Lieberman et al, 2003; Kaminski et al, 2014; Baccaro et al, 2015). A study from Germany showed that male sex and smoking had grater impact in the prevalence of colorectal lesions than family history of CRC (Hoffmeister et al, 2010). However, these studies analysed either a study population of unequal gender distribution (100\% women (Baccaro et al, 2015) and 97\% men (Lieberman et al, 2003)), focused on advanced lesions only (Kaminski et al, 2014) or included a smaller patient population (Hoffmeister et al, 2010). A major finding of the present study was to confirm smoking, as the strongest modifiable risk factor for both adenomas and advanced adenomas for both sexes. In addition, we showed in a large screening cohort with an almost equal distribution of men and women that the association was significantly stronger for women. A study on 1996 individuals investigated the impact of exposure level of smoking, and revealed that female smokers had an increase risk for advanced adenomas compared with non-smokers at a lower exposure level than man (Anderson et al, 2011). Exposure levels were not assed in the present study.

The correlation of BMI, colorectal neoplasia and sex is discussed controversially in the literature. Several colonoscopybased studies found a higher risk for colorectal lesions in women.
However, these studies were limited to a small patient count (Anderson et al, 2007; Stein et al, 2010) or a population of 97\% men (Lieberman et al, 2003). Conversely, a meta-analysis showed that the association between increased BMI and colon cancer was stronger in men than in women (Larsson and Wolk, 2007). Interestingly, the sex difference in risk for CRC was smaller when waist circumference was measured (Larsson and Wolk, 2007) and the risk for adenomas is even similar, when waist circumference or waist to hip ration was measured (Hong et al, 2012). In the present study, higher BMI was significantly associated with higher rates of adenomas and advanced adenomas in both men and women. Waist circumference and waist to hip ration were not analysed in this study. As obesity is a strong risk factor for diabetes, correspondingly presence of diabetes was also associated with higher prevalence rates of both adenomas and advanced adenomas in both sexes; concordant to results of a meta-analysis on the correlation between diabetes and risk for CRC (Larsson et al, 2005). Also this study confirms the results of a smaller screening cohort, suggesting that increasing blood glucose levels are significantly associated with colorectal adenomas (Rampal et al, 2014).

Interestingly, high alcohol intake only increased the incidence of advanced adenomas in men. It neither affected 
Table 4. Multivariable models for adenoma and advanced adenoma

\begin{tabular}{|c|c|c|c|}
\hline Variable/risk factor & Group & $\begin{array}{c}\text { Adenomas } \\
\text { Odds ratio } \\
(95 \% \mathrm{Cl})\end{array}$ & $\begin{array}{c}\text { Advanced } \\
\text { adenomas } \\
\text { Odds ratio } \\
(95 \% \mathrm{Cl})\end{array}$ \\
\hline Age & $\begin{array}{l}>60 \\
\leqslant 0 \text { (Ref.) }\end{array}$ & $\begin{array}{c}1.84(1.69,2.00) \\
1\end{array}$ & $\begin{array}{c}2.11(1.72,2.5) \\
1\end{array}$ \\
\hline Gender & $\begin{array}{l}\text { M } \\
F(\text { Ref.) }\end{array}$ & $\begin{array}{c}1.96(1.81,2.10) \\
1\end{array}$ & $\begin{array}{c}1.91(1.58,2.32) \\
1\end{array}$ \\
\hline Triglycerides & $\begin{array}{l}>107 \\
\leqslant 07 \text { (Ref.) }\end{array}$ & $\begin{array}{c}1.03(1.00,1.06) \\
1\end{array}$ & - \\
\hline BMI & $\begin{array}{l}>26.3 \\
\leqslant 26.3 \text { (Ref.) }\end{array}$ & $\begin{array}{c}1.35(1.25,1.47) \\
1\end{array}$ & - \\
\hline Diastolic blood pressure & $\begin{array}{l}>80 \\
\leqslant 80 \text { (Ref.) }\end{array}$ & $\begin{array}{c}1.02(0.94,1.12) \\
1\end{array}$ & - \\
\hline Smoking (females) & $\begin{array}{l}\text { Yes } \\
\text { No (Ref.) }\end{array}$ & $\begin{array}{c}1.76(1.53,2.02) \\
1\end{array}$ & $\begin{array}{c}2.08(1.52,2.83) \\
1\end{array}$ \\
\hline Smoking (males) & $\begin{array}{l}\text { Yes } \\
\text { No (Ref.) }\end{array}$ & $\begin{array}{c}1.46(1.29,1.64) \\
1\end{array}$ & $\begin{array}{c}1.06(0.80,1.42) \\
1\end{array}$ \\
\hline Physical activity & $\begin{array}{l}\text { None } \\
\text { Occasionally } \\
\text { Regularly } \\
\text { (Ref.) }\end{array}$ & $\begin{array}{l}- \\
- \\
-\end{array}$ & $\begin{array}{c}1.54(1.18,2.00) \\
1.17(1.00,1.38) \\
1\end{array}$ \\
\hline GGT & $\begin{array}{l}>25 \\
\leqslant 5 \text { (Ref.) }\end{array}$ & - & $\begin{array}{c}1.05(0.85,1.29) \\
1\end{array}$ \\
\hline Cholesterol level & $\begin{array}{l}>217 \\
\leqslant 217 \text { (Ref.) }\end{array}$ & - & $\begin{array}{c}1.13(1.02,1.25) \\
1\end{array}$ \\
\hline Blood glucose level & $\begin{array}{l}>95 \\
\leqslant 95 \text { (Ref.) }\end{array}$ & - & $\begin{array}{c}1.05(1.01,1.09) \\
1\end{array}$ \\
\hline Alcohol score & $\begin{array}{l}\geqslant 2 \\
\leqslant 1 \text { (Ref.) }\end{array}$ & - & $\begin{array}{c}1.09(1.01,1.18) \\
1\end{array}$ \\
\hline
\end{tabular}

\begin{tabular}{|c|c|c|}
\hline Outcome & $\begin{array}{l}\text { Gender gap in } \\
\text { detection rates } \\
\text { (males-females) }\end{array}$ & $\begin{array}{c}\% \text { of gender gap } \\
\text { mediated by risk } \\
\text { factors }(95 \% \mathrm{Cl})\end{array}$ \\
\hline \multicolumn{3}{|c|}{ Adenoma detection rate } \\
\hline $\begin{array}{l}\text { Age-adjusted gender gap } \\
\text { Gender gap remaining } \\
\text { after adjustment for all } \\
\text { risk factors }{ }^{\mathrm{a}}\end{array}$ & $\begin{array}{l}10.4 \% \\
9.8 \%\end{array}$ & $\begin{array}{c}0 \% \\
8.6 \%(5.4-11.8 \%)\end{array}$ \\
\hline \multicolumn{3}{|c|}{ Advanced adenoma detection rate } \\
\hline $\begin{array}{l}\text { Age-adjusted gender gap } \\
\text { Gender gap remaining } \\
\text { after adjustment for all } \\
\text { risk factors }^{\mathrm{a}}\end{array}$ & $\begin{array}{l}3.17 \% \\
2.70 \%\end{array}$ & $\begin{array}{c}0 \% \\
14.2 \%(8.5-36.9 \%)\end{array}$ \\
\hline
\end{tabular}

advanced adenomas in women nor adenomas in both genders nor was high alcohol consumption an independent risk factor for colorectal lesions in a multivariate analysis. The association between high GGT levels and colorectal lesions might be explained by the association between non-alcoholic fatty liver disease (NAFLD) and colorectal lesions, since high GGT levels commonly occur in NAFLD (Hwang et al, 2010).

To estimate the impact of the investigated risk factors on the prevalence of premalignant colorectal lesions, we calculated PAFs. The results of this analysis revealed that unfavourable exposure to any of the independent risk factors was responsible for $16.5 \%$ of adenomas and $13.2 \%$ of advanced adenomas. To the authors' knowledge, this is so far the largest study investigating as much cardiovascular and lifestyle risk factors for premalignant colorectal lesions in an asymptomatic study population with almost equal gender distribution. By calculating PAFs, it was estimated that smokers had a $4.75 \%$ higher risk for adenomas and a $6.57 \%$ higher risk for advanced adenomas than nonsmokers. The PAFs for smoking in this study were comparable to a study conducted in Germany, which calculated a PAF for smoking on adenomas of $7 \%$ and for advanced adenomas of 9\% (Hoffmeister et al, 2010).

Several attempts have been made in the past to develop risk stratification approach in CRC screening (Colditz et al, 2000; Freedman et al, 2009; Park et al, 2009). Results of this study contribute significantly towards the concept of individualised screening approach, especially for the most adequate approach for one-time colonoscopic screening to determine the most cost effective approach in screening colonoscopy (Ness et al, 2000). Screening colonoscopy is considered the gold standard in CRC prevention, if required benchmark quality parameters are met. However, in times of limited financial resources and screening colonoscopy is an invasive examination on potentially healthy screening individuals, current EU guidelines recommend faecal immunohistochemical stool test (FIT) as alternative to screening colonoscopy(Segnan et al, 2010). However FOBT/FIT are almost insensitive on sessile-serrated adenomas/polyps (SSA/P), which are the likely precursor lesions of $20-30 \%$ of $\mathrm{CRC}$, as those lesion do not bleed. FIT has a point sensitivity of $46 \%$ for high-grade dysplasia and 5\% for SSA/P, whereas the multi-target stool DNA test has $42 \%$ sensitivity for SSA/P $1 \mathrm{~cm}$ in size or greater and $69 \%$ sensitivity for high-grade dysplasia. To take advantage of the latency of CRC precursors to prevent CRC development through screening, the choice of invasive and non-invasive tests to use are critical to determining a program's effectiveness (Imeriale et al, 2014).

Strengths of this study are the large cohort of a consecutive series of asymptomatic screening individuals, as well as the large number of investigated risk factors. Another strengths is the almost equal proportion of women $(50.8 \%)$ and men; most prior studies include mostly men, therefore results of these studies are not generalisable for screening cohorts and thus subsequently for screening recommendations.

This study has some limitations to be acknowledged. A study limitation was that we did not assess family history of cancer, hormone replacement therapy, red meat or fibre intake. Another limitation of this study was that the overall ADR of $19.4 \%$ is lower than the current recommended benchmark of $25 \%$ (Rex et al, 2015) and lower than the previous benchmark of $20 \%$ (Rex et al, 2006). A recent analysis of the present study cohort, however, revealed that although ADR at the time of implementation of the quality assurance program for screening colonoscopy was below quality benchmarks, detection rates showed a positive trend in the following years. The authors conclude that this positive trend might result from a constant audit and feedback within the quality assurance program, as well as a general higher level of awareness regarding the impact and importance of high-quality performance in screening colonoscopy (Waldmann et al, 2016).

In summary, smoking was the only sex-specific-independent risk factor for precancerous colorectal lesions, affecting women stronger than men. Several risk factors were shown to impact either men or women or both sexes. However, the influence of risk factors only explained $0.6 \%$ of the $10.4 \%$ gender difference in adenoma detection and $0.47 \%$ of the $3.17 \%$ difference in advanced adenoma detection rate revealing male sex as the strongest risk factor for colorectal lesions. 
Table 6. Population attributable fractions (PAF): proportion of detected adenomas and advanced adenomas that are attributable to unfavourable risk factor exposure

\begin{tabular}{|c|c|c|c|c|c|c|c|}
\hline Risk factor & $\begin{array}{l}\text { Males, } \\
\text { reference }\end{array}$ & $\begin{array}{c}\text { Males, } N(\%) \\
\text { outside } \\
\text { reference }\end{array}$ & $\begin{array}{l}\text { Females, } \\
\text { reference }\end{array}$ & $\begin{array}{c}\text { Females, } N(\%) \\
\text { outside } \\
\text { reference }\end{array}$ & $\begin{array}{c}\text { Total, } \mathbf{N}(\%) \\
\text { outside } \\
\text { reference }\end{array}$ & $\begin{array}{l}\text { PAF adenomas } \\
(95 \% \mathrm{Cl})\end{array}$ & $\begin{array}{c}\text { PAF advanced } \\
\text { adenomas } \\
(95 \% \mathrm{Cl})\end{array}$ \\
\hline GGT & $<60$ & 2175 (17.4\%) & $<40$ & $1698(13.2 \%)$ & $3873(15.3 \%)$ & $0.48 \%(-0.22$ to $1.18 \%)$ & $0.81 \%(-0.79$ to $2.41 \%)$ \\
\hline $\mathrm{BMI}$ & $23-30$ & $3547(29.1 \%)$ & $23-30$ & $5586(44.3 \%)$ & $9133(36.8 \%)$ & $0.87 \%(-0.17$ to $1.91 \%)$ & $-0.44 \%(-2.43$ to $1.54 \%)$ \\
\hline Cholesterol & $<200$ & $4870(39.0 \%)$ & $<200$ & $6011(46.6 \%)$ & 16802 (66.2\%) & $1.81 \%(-0.59$ to $4.21 \%)$ & $3.42 \%(-1.51$ to $8.36 \%)$ \\
\hline HDL cholesterol & $>55$ & $7312(58.5 \%)$ & $>65$ & $6591(51.1 \%)$ & $13903(54.7 \%)$ & $2.94 \%(1.21-4.67 \%)$ & $6.05 \%(2.69-9.42 \%)$ \\
\hline Triglycerides & $<150$ & $3848(31.1 \%)$ & $<150$ & $2540(19.9 \%)$ & $6388(25.4 \%)$ & $1.32 \%(0.60-2.04 \%)$ & $2.99 \%(1.50-4.49 \%)$ \\
\hline $\begin{array}{l}\text { Systolic blood } \\
\text { pressure }\end{array}$ & $<140$ & $3688(29.5 \%)$ & $<140$ & 3395 (26.3\%) & 7083 (27.9\%) & $1.91 \%(0.90$ to $2.91 \%)$ & $2.70 \%(0.31-5.08 \%)$ \\
\hline $\begin{array}{l}\text { Diastolic blood } \\
\text { pressure }\end{array}$ & $<90$ & $1800(14.4 \%)$ & $<90$ & $1530(11.9 \%)$ & $3330(13.1 \%)$ & $1.68 \%(0.87-2.49 \%)$ & $1.58 \%(0.77-2.38 \%)$ \\
\hline Alcohol score & $0-7$ & $178(2.8 \%)$ & $0-4$ & $128(2.0 \%)$ & $306(2.4 \%)$ & $0.03 \%(-0.09$ to $0.15 \%)$ & $0.36 \%(0.00-0.70 \%)$ \\
\hline $\begin{array}{l}\text { Blood glucose } \\
\text { level }\end{array}$ & 74-109 & $3123(25.0 \%)$ & 74-109 & $2406(18.6 \%)$ & 5529 (21.8\%) & $0.62 \%(0.07-1.17 \%)$ & $0.70 \%(-0.43$ to $1.82 \%)$ \\
\hline $\begin{array}{l}\text { New Zealand } \\
\text { cardiovascular } \\
\text { risk score }\end{array}$ & $1-4$ & 2834 (22.7\%) & $1-4$ & 649 (5.0\%) & $3483(13.7 \%)$ & $3.15 \%(2.43-3.87 \%)$ & $5.72 \%(3.88-7.57 \%)$ \\
\hline Physical activity & $\begin{array}{l}\text { Occasionally } \\
\text { or regularly }\end{array}$ & $883(7.1 \%)$ & $\begin{array}{l}\text { Occasionally } \\
\text { or regularly }\end{array}$ & $1044(8.1 \%)$ & $1927(7.6 \%)$ & $0.76 \%(-0.01$ to $1.53 \%)$ & $2.66 \%(0.95-4.37 \%)$ \\
\hline Diabetes mellitus & no & $1070(8.6 \%)$ & no & $743(5.8 \%)$ & $1813(7.1 \%)$ & $1.10 \%(0.32-1.89 \%)$ & $2.92 \%(1.16-4.68 \%)$ \\
\hline Smoking & no & $1706(13.6 \%)$ & no & $1687(13.1 \%)$ & $3393(13.4 \%)$ & $4.75 \%(3.66-5.84 \%)$ & $6.37 \%(4.16-8.59 \%)$ \\
\hline $\begin{array}{l}\text { All risk factors } \\
\text { (multivariable } \\
\text { model) }\end{array}$ & & $10063(81.7 \%)$ & & $8843(69.7 \%)$ & $18906(75.6 \%)$ & $16.7 \%(13.9-19.4 \%)$ & $13.2 \%(8.1-18.2 \%)$ \\
\hline
\end{tabular}

\section{ACKNOWLEDGEMENTS}

This study was supported by the Skoda Award of the Austrian Society of Internal Medicine (to MF). This study was IRB approved (EK 1417/2014) by the ethics committee of the Medical University of Vienna.

\section{CONFLICT OF INTEREST}

Data on screening colonoscopy stem from the 'Quality certificate for CRC prevention' founded by the Austrian Society for Gastroenterology and Hepatology, the Federation of the Statutory Insurance Institutions and the Austrian Cancer Aid. This study was further supported by the Skoda Award of the Austrian Society of Internal Medicine (to MF).

\section{AUTHOR CONTRIBUTIONS}

Elisabeth Waldmann: study concept and design; acquisition of data; analysis and interpretation of data; drafting of the manuscript; and critical revision of the manuscript for important intellectual content. Georg Heinze: statistical analysis; analysis and interpretation of data; and critical revision of the manuscript for important intellectual content. Arnulf Ferlitsch: study concept and design; and critical revision of the manuscript for important intellectual content. Irina Gessl: acquisition of data. Daniela Sallinger: acquisition of data. Philip Jeschek: critical revision of the manuscript for important intellectual content. Martha BrittoArias: acquisition of data; and technical support. Petra Salzl: acquisition of data; and study concept and design. Elisabeth
Fasching: study concept and design; acquisition of data; and technical support. Bernd Jilma: analysis and interpretation of data; and critical revision of the manuscript for important intellectual content. Michael Kundi: analysis and interpretation of data; and critical revision of the manuscript for important intellectual content. Michael Trauner: critical revision of the manuscript for important intellectual content. Monika Ferlitsch: study concept and design; acquisition of data; analysis and interpretation of data; critical revision of the manuscript for important intellectual content; obtained funding; and study supervision.

\section{REFERENCES}

Anderson JC, Messina CR, Dakhllalah F, Abraham B, Alpern Z, Martin C, Hubbard PM, Grimson R, Shaw RD (2007) Body mass index: a marker for significant colorectal neoplasia in a screening population. J Clin Gastroenterol 41(3): 285-290.

Anderson JC, Moezardalan K, Messina CR, Latreille M, Shaw RD (2011) Smoking and the association of advanced colorectal neoplasia in an asymptomatic average risk population: analysis of exposure and anatomical location in men and women. Dig Dis Sci 56(12): 3616-3623.

Babor TF, Higgins-Biddle JC, Saunders JB, Monteiro MG (2001) AUDIT. The Alcohol Use Disorders Identification Test. In Guidelines for Use in Primary Care, 2nd edn. World Health Organization: Geneva. Available at http://apps.who.int/iris/bitstream/10665/67205/1/ WHO_MSD_MSB_01.6a.pdf.

Baccaro LF, Conde DM, Costa-Paiva L, de Souza Santos Machado V, Pinto-Neto AM (2015) Cancer in women over 50 years of age: a focus on smoking. Cancers 7(1): 450-459.

Bannert C, Reinhart K, Dunkler D, Trauner M, Renner F, Knoflach P, Ferlitsch A, Weiss W, Ferlitsch M (2012) Sedation in screening colonoscopy: impact on quality indicators and complications. Am J Gastroenterol 107(12): 1837-1848. 
Ben Q, An W, Jiang Y, Zhan X, Du Y, Cai QC, Gao J, Li Z (2012) Body mass index increases risk for colorectal adenomas based on meta-analysis. Gastroenterology 142(4): 762-772.

Cho E, Smith-Warner SA, Ritz J, van den Brandt PA, Colditz GA, Folsom AR, Freudenheim JL, Giovannucci E, Goldbohm RA, Graham S, Holmberg L, Kim DH, Malila N, Miller AB, Pietinen P, Rohan TE, Sellers TA, Speizer FE, Willett WC, Wolk A, Hunter DJ (2004) Alcohol intake and colorectal cancer: a pooled analysis of 8 cohort studies. Ann Intern Med 140(8): 603-613.

Colditz GA, Atwood KA, Emmons K, Monson RR, Willett WC, Trichopoulos D, Hunter DJ (2000) Harvard report on cancer prevention volume 4: Harvard Cancer Risk Index. Risk Index Working Group, Harvard Center for Cancer Prevention. Cancer Causes Control 11(6): 477-488.

Ferlitsch M, Reinhart K, Pramhas S, Wiener C, Gal O, Bannert C, Hassler M, Kozbial K, Dunkler D, Trauner M, Weiss W (2011) Sex-specific prevalence of adenomas, advanced adenomas, and colorectal cancer in individuals undergoing screening colonoscopy. JAMA 306(12): 1352-1358.

Freedman AN, Slattery ML, Ballard-Barbash R, Willis G, Cann BJ, Pee D, Gail MH, Pfeiffer RM (2009) Colorectal cancer risk prediction tool for white men and women without known susceptibility. J Clin Oncol 27(5): 686-693.

Hermann S, Rohrmann S, Linseisen J (2009) Lifestyle factors, obesity and the risk of colorectal adenomas in EPIC-Heidelberg. Cancer Causes Control 20(8): $1397-1408$

Hoffmeister M, Schmitz S, Karmrodt E, Stegmaier C, Haug U, Arndt V, Brenner H (2010) Male sex and smoking have a larger impact on the prevalence of colorectal neoplasia than family history of colorectal cancer. Clin Gastroenterol Hepatol 8(10): 870-876.

Hong S, Cai Q, Chen D, Zhu W, Huang W, Li Z (2012) Abdominal obesity and the risk of colorectal adenoma: a meta-analysis of observational studies. Eur J Cancer Prev 21(6): 523-531.

Hwang ST, Cho YK, Park JH, Kim HJ, Park DI, Sohn CI, Jeon WK, Kim BI, Won KH, Jin W (2010) Relationship of non-alcoholic fatty liver disease to colorectal adenomatous polyps. J Gastroenterol Hepatol 25(3): 562-567.

Imeriale TF, Ranshoff DF, Itzkowitz SH (2014) Multitarget stool DNA testing for colorectal-cancer screening. N Engl J Med 371(2): 187-188.

Kaminski MF, Polkowski M, Kraszewska E, Rupinski M, Butruk E, Regula J (2014) A score to estimate the likelihood of detecting advanced colorectal neoplasia at colonoscopy. Gut 63(7): 1112-1119.

Kozbial K, Reinhart K, Heinze G, Zwatz C, Bannert C, Salzl P, Waldmann E, Britto-Arias M, Ferlitsch A, Trauner M, Weiss W, Ferlitsch M (2014) High quality of screening colonoscopy in Austria is not dependent on endoscopist specialty or setting. Endoscopy 47(3): 207-216.

Larsson SC, Orsini N, Wolk A (2005) Diabetes mellitus and risk of colorectal cancer: a meta-analysis. J Natl Cancer Inst 97(22): 1679-1687.

Larsson SC, Wolk A (2007) Obesity and colon and rectal cancer risk: a metaanalysis of prospective studies. Am J Clin Nutr 86(3): 556-565.

Lieberman DA, Prindiville S, Weiss DG, Willett W. VA Cooperative Study Group 380 (2003) Risk factors for advanced colonic neoplasia and hyperplastic polyps in asymptomatic individuals. JAMA 290(22): 2959-2967.

Ness RM, Holmes AM, Klein R, Dittus R (2000) Cost-utility of one-time colonoscopic screening for colorectal cancer at various ages. Am J Gastroenterol 95(7): 1800-1811.

New Zealand Primary Care Handbook 2012. (updated 2013) Cardiovascular Disease Risk Assessment. Available at http://www.health.govt.nz/system/ files/documents/publications/cardiovascular-disease-risk-assessmentupdated-2013-dec13.pdf.

Nguyen SP, Bent S, Chen YH, Terdiman JP (2009) Gender as a risk factor for advanced neoplasia and colorectal cancer: a systematic review and metaanalysis. Clin Gastroenterol Hepatol 7(6): 676-81 e1-3.

NHLBI Obesity Education Initiative (2000) The Practical Guide. Identification, Evaluation and Treatment of Overweight and Obesity in Adults.

Park Y, Freedman AN, Gail MH, Pee D, Hollenbeck A, Schatzkin A, Pfeiffer RM (2009) Validation of a colorectal cancer risk prediction model among white patients age 50 years and older. J Clin Oncol 27(5): 694-698.

Rampal S, Yang MH, Sung J, Son HJ, Choi YH, Lee JH, Kim YH, Chang DK, Rhee PL, Rhee JC, Guallar E, Cho J (2014) Association between markers of glucose metabolism and risk of colorectal adenoma. Gastroenterology 147(1): 78-87 e3.

Regula J, Rupinski M, Kraszewska E, Polkowski M, Pachlewski J, Orlowska J, Nowacki MP, Butruk E (2006) Colonoscopy in colorectal-cancer screening for detection of advanced neoplasia. N Engl J Med 355(18): 1863-1872.

Reinhart K, Bannert C, Dunkler D, Salzl P, Trauner M, Renner F, Knoflach P, Ferlitsch A, Weiss W, Ferlitsch M (2013) Prevalence of flat lesions in a large screening population and their role in colonoscopy quality improvement. Endoscopy 45(5): 350-356.

Rex DK, Petrini JL, Baron TH, Chak A, Cohen J, Deal SE, Hoffman B, Jacobson BC, Mergener K, Petersen BT, Safdi MA, Faigel DO, Pike IM. ASGE/ACG Taskforce on Quality in Endoscopy (2006) Quality indicators for colonoscopy. Am J Gastroenterol 101(4): 873-885.

Rex DK, Schoenfeld PS, Cohen J, Pike IM, Adler DG, Fennerty MB, Lieb 2nd JG, Park WG, Rizk MK, Sawhney MS, Shaheen NJ, Wani S, Weinberg DS (2015) Quality indicators for colonoscopy. Am J Gastroenterol 110(1): 72-90.

Schmiegel W, Reinacher-Schick A, Arnold D, Graeven U, Heinemann V, Porschen R, Riemann J, Rödel C, Sauer R, Wieser M, Schmitt W, Schmoll HJ, Seufferlein T, Kopp I, Pox C (2008) [Update S3-guideline "colorectal cancer" 2008]. Z Gastroenterol 46(8): 799-840.

Segnan N, Patnick J, von Karsa L (2010) European guidelines for quality assurance in colorectal cancer screening and diagnosis. Luxembourg: Publications Office of the European Union: European Commission.

Siegel R, Naishadham D, Jemal A (2013) Cancer statistics, 2013. CA Cancer J Clin 63(1): 11-30.

Stein B, Anderson JC, Rajapakse R, Alpern ZA, Messina CR, Walker G (2010) Body mass index as a predictor of colorectal neoplasia in ethnically diverse screening population. Dig Dis Sci 55(10): 2945-2952.

Thomas DB, Karagas MR (1987) Cancer in first and second generation Americans. Cancer Res 47(21): 5771-5776.

Waldmann E, Britto-Arias M, Gessl I, Heinze G, Salzl P, Sallinger D, Trauner M, Weiss W, Ferlitsch A, Ferlitsch M (2015) Endoscopists with low adenoma detection rates benefit from high-definition endoscopy. Surg Endosc 29(2): 466-473.

Waldmann E, Gessl I, Sallinger D, Jeschek P, Britto-Arias M, Heinze G, Fasching E, Weiss W, Gschwantler M, Trauner M, Ferlitsch M (2016) Trends in quality of screening colonoscopy in Austria. Endoscopy; e-pub ahead of print 30 August 2016.

This work is published under the standard license to publish agreement. After 12 months the work will become freely available and the license terms will switch to a Creative Commons AttributionNonCommercial-Share Alike 4.0 Unported License. 